\title{
Rural-Urban Differences in Health Care Expenditures: Empirical Data from US Households
}

\author{
Wei-Chen Lee, ${ }^{1}$ Luohua Jiang, ${ }^{2}$ Charles D. Phillips, ${ }^{3}$ and Robert L. Ohsfeldt ${ }^{3}$ \\ ${ }^{1}$ Center to Eliminate Health Disparities, University of Texas Medical Branch, Galveston, TX, USA \\ ${ }^{2}$ Department of Epidemiology and Biostatistics, School of Rural Public Health, Texas A\&M Health Science Center, \\ College Station, TX, USA \\ ${ }^{3}$ Department of Health Policy and Management, School of Rural Public Health, Texas A\&M Health Science Center, \\ College Station, TX, USA
}

Correspondence should be addressed to Robert L. Ohsfeldt; rohsfeldt@sph.tamhsc.edu

Received 31 May 2014; Revised 26 August 2014; Accepted 27 August 2014; Published 9 September 2014

Academic Editor: Shenying Fang

Copyright (C) 2014 Wei-Chen Lee et al. This is an open access article distributed under the Creative Commons Attribution License, which permits unrestricted use, distribution, and reproduction in any medium, provided the original work is properly cited.

\begin{abstract}
Purpose. To estimate the rural-urban differences in expenditures of outpatient care, hospital inpatient care, hospital emergency room services, medications, and total services. Methods. This cross-sectional study used data from the 2010 Medical Expenditure Panel Survey. The overall sample size for the study was 22,772 . Weighted frequencies, means, or percentages were estimated to illustrate the distribution of each variable. Five two-part utilization models were then fit to determine the likelihood of having nonzero expenses and to identify how residence in a rural versus urban area affected expenditures in our five expense categories. Quantile regressions were estimated to further explore relationships between residence and each quantile of nonzero expenditure. Results. The results of two-part model suggest that rural populations spent more on medications, while urban populations spent more on emergency care. However, no rural-urban difference was found in total health expenditures. The results of quantile regressions suggest that the highest users (at the upper quantiles) of medication and total expenditure experienced the strongest positive effects of living in rural areas. Conclusions. Total health expenditures do not seem to vary significantly across urban and rural areas. However, rurality does have important effects on those who make the most use of outpatient care and prescription medications. Reviewing total health expenditures for urban and rural populations is not enough. Policymakers should monitor the effects of geographic differences, especially in the highest expenditure quantiles, for specific types of health expenditures. Differences in the influence of rurality across this distribution of health expenditures may provide important guidance for interventions.
\end{abstract}

\section{Introduction}

The Center for Medicare \& Medicaid Services (CMS) reported that national health expenditures grew dramatically from $\$ 1493.3$ billion in 2001 to $\$ 2700.7$ billion in 2011 [1]. National health expenditures are now projected to reach $\$ 4,781.0$ billion in 2021 [2]. Hospital care, professional services, and prescription drugs are the three categories of health expenditures with the highest per capita figures [1]. On average, individuals spent $\$ 2,734$ on hospital care, $\$ 1,740$ on physician services (excluding dental services), and $\$ 845$ on prescription drugs in 2011.

The growth of healthcare expenditures is of particular concern to rural populations whose incomes are significantly lower than their urban counterparts [3]. This research examines rural-urban differences in total health care expenditures, as well as expenditures for different types of health services (i.e., outpatient care, hospital inpatient care, hospital emergency room services, and prescription drugs).

Data for health expenditures for individuals residing in urban or rural areas were obtained from the Medical Expenditure Panel Survey (MEPS). As a nationally representative data source, MEPS data are particularly well suited for the task of estimating rural-urban differences in health care expenditures [4]. Among prior studies using MEPS data, findings about differences in health expenditures between rural and urban populations have been quite mixed. Ziller and colleagues concluded that residents in rural areas had 
higher out-of-pocket spending on healthcare than those living in urban areas [5]. However, expenditures for dental care for older adults living in large metropolitan areas were higher than those in small metropolitan and nonmetropolitan areas [6]. On the other hand, Chevarley and colleagues pointed out that there were no geographic differences in healthcare expenditures for children [7]. Another study about veterans' healthcare expenditures concluded that rural veterans (VA) younger than 65 years spent $\$ 1,100$ less on average than urban VA users, but the average rural VA user aged 65 and older spent \$250 more than urban veterans [8].

This study extends existing research in two important ways. First, the study focuses on urban-rural differences in health spending for the four most costly categories. Second, in addition to using traditional two-part models to examine the relationship between the urban-rural residency and health expenditures, exploratory quantile regression models were used to assess the extent to which urban-rural differences vary across quantiles of the expenditure distribution. The latter may be important because a number of studies have reported an extraordinarily high concentration of healthcare costs and utilization among small segments of the population [9-12]. For example, 64 percent of total healthcare expenditures were accumulated by only 15 percent of patients [12].

\section{Methods}

2.1. Data Source. The cross-sectional data used in this study were drawn from a subsample of the 2010 Medical Expenditure Panel Survey (MEPS), a nationally representative survey of the US civilian noninstitutionalized population [13]. The subsample of individual household members consisted of households in the 2010 MEPS sample who also participated in the National Health Interview Survey (NHIS) in 2008 or 2009. The sampling plan of the NHIS followed a multistage area probability design but oversampled households with Blacks, Hispanics, Asians, and low income families to improve the precision of estimates for these selected subgroups [14]. People who were in the military, born abroad, institutionalized, or who died during the reference period were not eligible for this survey.

Like NHIS, the AHRQ used a multistage stratified sampling design [15]. The first stage consisted of a sample of 428 PSUs drawn from 1,900 geographically defined PSUs nationwide [16]. Each PSU contained a county, a small group of contiguous counties, or a metropolitan statistical area (MSA). The second stage sampling used either area segments or permit segments to draw survey samples. An area segment comprised about eight, twelve, or sixteen addresses. A permit segment covered housing units built after the 2000 census, which generally included four addresses.

This 2010 file contains the household component (HC) and the medical provider component (MPC). Individual characteristics such as gender were collected through computer assisted personal interviewing (CAPI) technology in the HC [13]. With permission from the household survey respondents, the MPC collected data about visits, diagnosis, charges, and payments from the healthcare providers of household members. The MPC data supplemented the MEPS household reported information on health expenditures.

Households selected through the stratified sampling approach were interviewed 5 times across two years. Data for the year 2010 came from rounds 3-5 of panel 14 (a subsample of the 2008 NHIS responding households) and rounds 13 of panel 15 (a subsample of the 2009 NHIS responding households) [15]. The response rate of panel 14 was $85.2 \%$ and $84.0 \%$ for panel 15 . The public use dataset pooled 18,398 families with 31,228 valid cases.

This study sample was limited to adults 18 years or older who completed health-related questions such as disease diagnoses. The final sample included 22,772 $(=n)$ adults that after the application of appropriate sampling weights represented $229,857,784(=N)$ adults in the US.

\subsection{Dependent Variables: Healthcare Expenditures. Unlike} the NHIS, the MEPS contained the healthcare expenditures reported by household members and medical providers served as dependent variables in this study [15]. Expenditures are the sum of out-of-pocket payments and payments by private insurance, Medicaid, Medicare, TRICARE, and other sources. In addition to total health expenditures, expenditures for four types of health services were chosen for this study: (1) expenses for outpatient care (for both hospitalbased and office-based providers of outpatient services), (2) expenditures for hospital inpatient care, (3) expenditures for hospital emergency room services, and (4) prescription drugs expenses.

Outpatient care data were provided by doctors practicing in either private clinics or hospital-based outpatient departments. Expenditures of hospital inpatient care and hospital emergency room services included both hospital facility expenses and payments for physicians whose inpatient services delivered in hospital settings. Expenditures for prescription medicines were obtained through both household interviews and pharmacy component surveys. Only prescription forms with valid fields for national drug code, medication name, strength of medicine (amount and unit), quantity (package size and amount), and payments by source were treated as valid cases. The last type of expenditure covered all services, including dental services and all other health services not included in our four subcategories of expenditures.

A traditional two-part model was used for expenditure data analysis. The first part of the two-part model focused on a dichotomous dependent variable indicating whether individuals had any expenditures in a particular service category (expenditure $=\$ 0$ or $>\$ 0$ ). The second part of the two-part model focused on the level of expenditures for individuals with nonzero expenditures in each of the service categories. Given skew in the distribution of expenditures, the level of nonzero health expenditures was transformed to the logarithmic scale for all of the expenditure categories.

AHRQ uses a hot-deck imputation process for missing data when both HC and MPC components were not collected or incomplete [15]. Regression models based on medical events with complete information were used to predict total 
expenses. Variables with known values such as total charge and provider type were used as predictors to form groups of donor events on expenditures. Then, a donor event with the closest predicted payment pattern was used to impute the missing values, taking into account the sampling weights associated with the MEPS complex survey design. Unfortunately, there is no variable in the MEPS data to flag which expenditure values were imputed, making it impossible to compare expenditures with and without imputation.

2.3. Independent Variables: Geographic Factor. The main independent variable of interest is an individual's place of residence ( $0=$ rural, $1=$ urban). Based on the 2000 report of Office of Management and Budget (OMB), urban areas in the MEPS refer to a metropolitan core based statistical area (CBSA), an area comprising at least one urbanized area that has a population of at least 50,000 [17]. The Office of Rural Health Policy (ORHP) defined all other areas as rural [18].

2.4. Covariates: Individual Characteristics. According to Andersen's model for individual use of health care $[19,20]$, this study used self-reported measures: (1) predisposing characteristics-age 18-44, 45-64, 65, and older, gender (female or male), race/ethnicity Hispanic, African American, White, or other, and highest level of education degree when the respondent first entered the study (degree lower than high school, high school, or higher than high school); (2) enabling resources-poverty status (poor or near poor, low income, middle income, or high income) and health insurance held (any private insurance, only public insurance, or uninsured); and (3) healthcare needs - the average perceived health status (very good or excellent, good, poor, or fair), average perceived mental health status (very good or excellent, good, poor, or fair), limitation in physical functioning (no limitation or any limitation), and presence of chronic diseases with relatively high prevalence (high blood pressure, heart diseases, stroke, emphysema, chronic bronchitis, high cholesterol, cancer, diabetes, joint pain, arthritis, and asthma). The functional limitation variable summarized whether a person had any activities of daily living (ADL), instrumental activities of daily living (IADL), or sensory limitations during any of the survey rounds [15].

2.5. Statistical Analyses. To reflect the complex survey design, the AHRQ used the Taylor-series linearization method to produce person-level variables for analysis, including perwt10f for sampling weight, varstr for strata, and varpsu for PSU [15]. Weighted frequencies, means, or percentages were estimated to illustrate the distribution of each variable. Correlations among independent variables were low enough $(r<0.75)$ to rule out multicollinearity.

Five two-part models were fit to the expenditure variables. In the first part, logistic regression models were used to determine the impact of urban-rural residency status on the likelihood of having nonzero expenditures (>\$0) in 2010 for each of the five expenditure categories. In the second part, regression models were used to assess the impact of urban-rural residency status on the natural logarithm of positive expenditure among individuals with positive healthcare expenditures for each of the five expenditure categories. In both parts, the models adjusted for the personal characteristics described in detail above: age, gender, race/ethnicity, educational attainment, poverty status, insurance status, perceived physical health status, perceived mental health status, limited physical activity, and a count of comorbid conditions.

Quantile regression models were then estimated to explore the relationships between urban or rural residency (for individuals with nonzero expenditures) at various quantiles of the nonzero expenditure distribution, adjusting for personal characteristics covariates. Taking into account the survey design, the bootstrapping method was used to draw an alternative sampling weight as well as to obtain standard errors without assumption. The regression coefficient at a given quantile indicates the effect of residence (i.e., rural or urban) on a unit change in that expenditure variable, assuming that the other variables are fixed, with 95\% confidence interval bands. Two-tailed $P$ values less than or equal to 0.05 were considered statistically significant. The goodness of fit was first examined by fitting the design-based model (i.e., takes the survey design structure into account), then estimating the corresponding probabilities, and subsequently using independently and identically distributed- (i.i.d.-) based tests. All data analyses were performed using Stata 13 using the "svy" procedure to incorporate survey sampling weights [21].

\section{Results}

Table 1 provides descriptive comparisons of weighted mean healthcare expenditures for persons living in rural or urban areas, as well as standard errors (SE), percentage with zero expenditures, and $P$ values for bivariate tests for any urban/rural differences. Overall, $15.8 \%$ of the weighted sample was from rural areas.

Rural populations spent more money on prescription drugs than urban populations (urban: \$1061.4; rural: \$1278.3; $P=0.007)$. After excluding zero users, urban populations (\$1636.4) spent more than rural populations (\$1167.4) on emergency room services $(P=0.0011)$. Next, there were higher proportions of zero users in rural areas than in urban areas in terms of emergency care, prescription drugs, and all services received $(P<0.052)$.

Focusing on the cumulative distribution of nonzero expenditures, the results indicate that in both urban and rural areas a small percentage of people accounted for a relatively large percentage of healthcare expenditures. For instance, less than $2 \%$ of rural or urban populations accounted for half of hospital inpatient care and emergency room service expenditures. In these two areas, expenditures were slightly more concentrated in urban versus rural areas.

Table 2 provides the weighted percentages and $P$ values for the personal characteristic covariates across rural and urban populations. Due to the large sample size, $P$ values for hypothesis tests of the null of no difference in means or proportions tend to be small even when the absolute differences in point estimates means or proportions are not large. On average, rural populations are more likely to be old 
TABLE 1: Comparisons of weighted individual expenditure distributions by residence and type of service, MEPS 2010.

\begin{tabular}{|c|c|c|c|}
\hline Weighted mean (SE) or percentage & $\begin{array}{c}\text { Urban } \\
(N=19,561)\end{array}$ & $\begin{array}{c}\text { Rural } \\
(N=3,211)\end{array}$ & $P$ value \\
\hline \multicolumn{4}{|l|}{ Outpatient care } \\
\hline Include zero (\$) & $1252.7(43.3)$ & $1306.2(62.2)$ & 0.4826 \\
\hline Exclude zero (\$) & $1852.5(60.7)$ & $1893.5(81.6)$ & 0.6854 \\
\hline Zero users (\%) & $32.4 \%$ & $31.0 \%$ & 0.5458 \\
\hline Lower half of cumulative sum (\%) & $63.1 \%$ & $64.2 \%$ & \\
\hline Higher half of cumulative sum (\%) & $4.5 \%$ & $4.8 \%$ & \\
\hline \multicolumn{4}{|l|}{ Hospital inpatient care } \\
\hline Include zero (\$) & $1602.8(90.8)$ & $1574.7(160.4)$ & 0.8775 \\
\hline Exclude zero (\$) & $18838.5(853.9)$ & $15747.0(1293.7)$ & 0.0538 \\
\hline Zero users (\%) & $91.5 \%$ & $90.0 \%$ & 0.0853 \\
\hline Lower half of cumulative sum (\%) & $7.3 \%$ & $8.3 \%$ & \\
\hline Higher half of cumulative sum (\%) & $1.2 \%$ & $1.7 \%$ & \\
\hline \multicolumn{4}{|l|}{ Hospital emergency room } \\
\hline Include zero (\$) & $187.1(9.8)$ & $163.4(19.1)$ & 0.2870 \\
\hline Exclude zero (\$) & $1636.4(76.4)$ & $1167.4(113.0)$ & 0.0011 \\
\hline Zero users $(\%)$ & $88.6 \%$ & $86.0 \%$ & 0.0087 \\
\hline Lower half of cumulative sum (\%) & $10.4 \%$ & $12.5 \%$ & \\
\hline Higher half of cumulative sum (\%) & $1.1 \%$ & $1.5 \%$ & \\
\hline \multicolumn{4}{|l|}{ Prescription drugs } \\
\hline Include zero $(\$)$ & $1061.4(31.0)$ & $1278.3(71.7)$ & 0.0073 \\
\hline Exclude zero (\$) & $1611.5(43.8)$ & $1741.7(93.6)$ & 0.2187 \\
\hline Zero users $(\%)$ & $34.1 \%$ & $26.6 \%$ & $<0.0001$ \\
\hline Lower half of cumulative sum (\%) & $60.9 \%$ & $66.3 \%$ & \\
\hline Higher half of cumulative sum (\%) & $4.9 \%$ & $7.0 \%$ & \\
\hline \multicolumn{4}{|l|}{ Total expenditures } \\
\hline Include zero (\$) & $4929.5(123.1)$ & $5172.3(269.2)$ & 0.4136 \\
\hline Exclude zero (\$) & $5867.1(143.2)$ & $6007.2(307.6)$ & 0.6788 \\
\hline Zero users (\%) & $16.0 \%$ & $13.9 \%$ & 0.0521 \\
\hline Lower half of cumulative sum (\%) & $78.3 \%$ & $79.3 \%$ & \\
\hline Higher half of cumulative sum (\%) & $5.7 \%$ & $6.8 \%$ & \\
\hline
\end{tabular}

Note: the last item "total expenditures" is not the sum of the above four services but the overall healthcare expenditure of each individual who might also use other services such as dental care.

$(P<0.0001)$, white $(P<0.0001)$, less educated $(P<0.0001)$, and poor $(P<0.0001)$, more likely to rely on public insurance $(P=0.004)$, to perceive poorer physical $(P<0.0001)$ and mental health status $(P=0.002)$, to have physical limitations $(P<0.0001)$, and to have multiple chronic diseases $(P<$ $0.0001)$.

Table 3 reports the results of two-part models by weighted coefficients for the urban (versus rural) residency variable, and the associated confidence intervals and $P$ values. The tests of goodness-of-fit suggest no evidence of lack of fit. In these logistic regression models that serve as the first part of the two-part model, urban residents were less likely to have zero expenditure for prescription drugs, compared to rural residents $(P=0.012)$. The estimated odds-ratio is 0.80 , which indicates that urban residents were $20 \%$ less likely to have zero prescription drug expenditure. Results for the residency variable in the other models indicate very small differences that do not approach statistical significance.
In the linear regression models that constitute the second part of the two-part model, urban residents displayed higher levels of expenditures for emergency services $(P=0.011)$. The estimated impact of urban residency is a 0.22 increase in conditional log-emergency-care-expenditure $(\beta=0.22)$, compared to rural populations.

Quantile regression models facilitate analysis of the full conditional distributional characteristics of the outcomes variables and yield estimates for the 5 conditional quantiles of expenditure given rurality (Figures 1, 2, 3, 4, and 5). In each figure, the solid line represents the estimated median effect and the dashed lines represent the associated confidence interval for the residency (urban) coefficient from the second part of the two-part model (reported in Table 3). If the solid line stays going down, it means living in rural areas is associated with the high expenditures. For example, the solid line descends toward increasing dollar values beginning around the 75th quantile in both Figures 4 and 5, though 
TABLE 2: Weighted description of personal characteristics by residence, MEPS 2010.

\begin{tabular}{|c|c|c|c|}
\hline Weighted percentage & $\begin{array}{c}\text { Urban } \\
(N=19,561)\end{array}$ & $\begin{array}{c}\text { Rural } \\
(N=3,211) \\
\end{array}$ & $P$ value \\
\hline \multicolumn{4}{|l|}{ Predisposing } \\
\hline \multicolumn{4}{|l|}{ Age } \\
\hline $18-44$ & 49.0 & 43.0 & \multirow{3}{*}{0.0003} \\
\hline $45-64$ & 34.8 & 36.7 & \\
\hline 65 and older & 16.2 & 20.3 & \\
\hline Gender (\% of women) & 51.5 & 51.9 & 0.6206 \\
\hline \multicolumn{4}{|l|}{ Race/ethnicity } \\
\hline Others & 7.3 & 3.3 & \multirow{4}{*}{$<0.0001$} \\
\hline Hispanic & 15.4 & 6.4 & \\
\hline Non-Hispanic black & 12.2 & 7.6 & \\
\hline Non-Hispanic white & 65.1 & 82.6 & \\
\hline \multicolumn{4}{|l|}{ Education level } \\
\hline Lower than high school & 16.5 & 22.8 & \multirow{3}{*}{$<0.0001$} \\
\hline Equal to high school & 53.3 & 58.0 & \\
\hline Higher than high school & 30.2 & 19.2 & \\
\hline \multicolumn{4}{|l|}{ Enabling } \\
\hline \multicolumn{4}{|l|}{ Poverty } \\
\hline Poor or near poor & 16.5 & 20.4 & \multirow{4}{*}{$<0.0001$} \\
\hline Low income & 13.0 & 15.3 & \\
\hline Middle income & 29.5 & 34.5 & \\
\hline High income & 41.0 & 29.8 & \\
\hline \multicolumn{4}{|l|}{ Health insurance status } \\
\hline Any private insurance & 68.0 & 63.9 & \multirow{3}{*}{0.0039} \\
\hline Only public insurance & 16.6 & 21.0 & \\
\hline Uninsured & 15.4 & 15.1 & \\
\hline \multicolumn{4}{|l|}{ Care needs } \\
\hline \multicolumn{4}{|l|}{ Perceived physical health status } \\
\hline Very good or excellent & 59.1 & 53.2 & \multirow{3}{*}{0.0001} \\
\hline Good & 27.0 & 29.1 & \\
\hline Poor or fair & 13.9 & 17.7 & \\
\hline \multicolumn{4}{|l|}{ Perceived mental health status } \\
\hline Very good or excellent & 70.0 & 65.0 & \multirow{3}{*}{0.0023} \\
\hline Good & 22.9 & 26.6 & \\
\hline Poor or fair & 7.1 & 8.3 & \\
\hline Any limitation on functions (\% of having any limitation) & 25.3 & 33.3 & $<0.0001$ \\
\hline \multicolumn{4}{|l|}{ Number of chronic diseases } \\
\hline No chronic disease & 35.7 & 30.3 & \multirow{3}{*}{$<0.0001$} \\
\hline 1 chronic disease & 21.9 & 19.3 & \\
\hline 2 and more chronic diseases & 42.4 & 50.4 & \\
\hline
\end{tabular}

the effect is not statistically significant. By contrast, rural residents had less expenditure on emergency room and the effect is statistically different from zero at very high quantiles (Figure 3). The coefficient for rurality grows from $\$ 318.0$ at the 0.75 th quantile to $\$ 1682.6$ at the 0.95 th quantile $(P<0.05)$.

\section{Discussion}

4.1. Comparisons of Healthcare Expenditures between Rural and Urban Areas. This study compared urban and rural populations with respect to their medical expenditures. To deal with high frequencies of zero expenditure, this study used 
TABLE 3: Two-part model estimated of impact of urban (versus rural) residency, weighted data adjusted for personal characteristics covariates, MEPS 2010.

\begin{tabular}{lcr}
\hline & O.R. (95\% C.I.) & $P$ value \\
\hline First-part (logistic regression) & & \\
Outpatient care & $1.086(0.944,1.249)$ & 0.246 \\
Hospital inpatient care & $0.943(0.783,1.138)$ & 0.542 \\
Hospital emergency room & $0.933(0.787,1.106)$ & 0.422 \\
Prescription drugs & $0.801(0.673,0.953)$ & 0.012 \\
Total expenditures & $1.020(0.830,1.252)$ & 0.853 \\
\hline & $\beta(95 \%$ C.I. $)$ & \\
\hline Second part (linear regression) & & 0.521 \\
Outpatient care & $0.027(-0.056 \sim 0.111)$ & 0.659 \\
Hospital inpatient care & $0.050(-0.174 \sim 0.274)$ & 0.011 \\
Hospital emergency room & $0.217(0.050 \sim 0.385)$ & 0.678 \\
Prescription drugs & $0.023(-0.087 \sim .133)$ & 0.634 \\
Total expenditures & $0.018(-0.058 \sim 0.095)$ & \\
\hline
\end{tabular}

Note: C.I.-confidence interval. Personal characteristics covariates: age, gender, race/ethnicity, educational attainment, poverty status, insurance status, perceived physical health status, perceived mental health status, functional limitations, and a count of comorbid conditions.

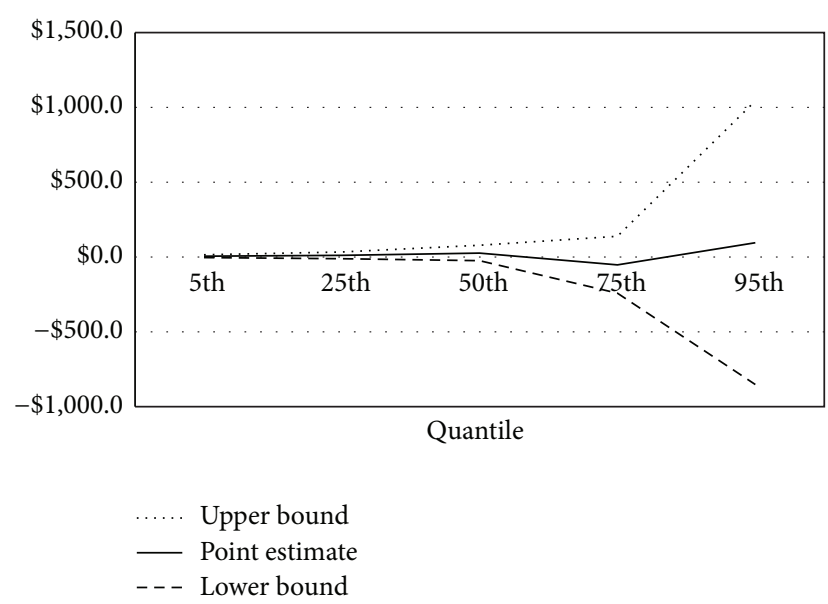

FIGURE 1: Weighted relationship between residence and nonzero expenditure of outpatient care, adjusted by 10 covariates.

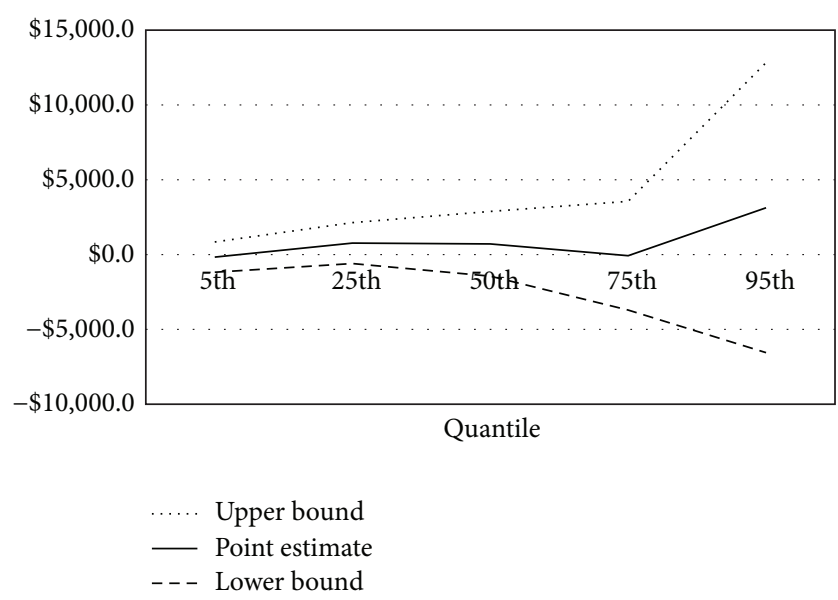

FIGURE 2: Weighted relationships between residence and nonzero expenditure of hospital inpatient care, adjusted by 10 covariates.

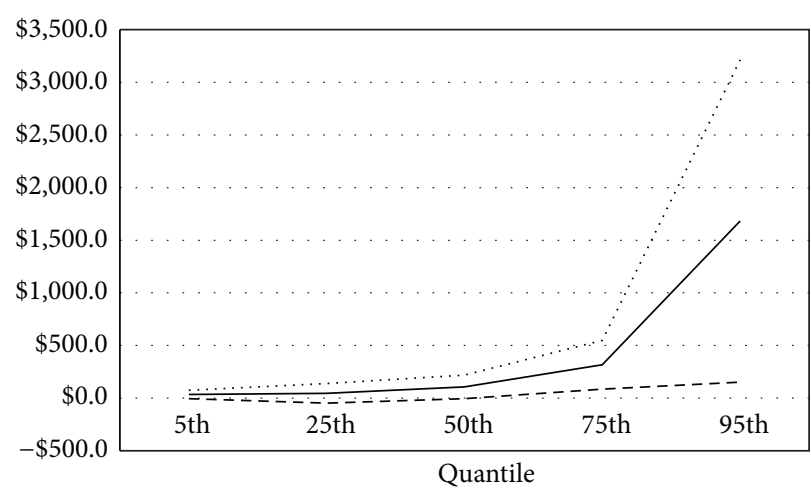

$$
\begin{aligned}
& \text { … Upper bound } \\
& \text { _ Point estimate } \\
& \text { _. - Lower bound }
\end{aligned}
$$

FIGURE 3: Weighted relationships between residence and nonzero expenditure of hospital emergency room service, adjusted by 10 covariates.

both two-part models and quantile regression models with adjustment for differences in a variety of personal characteristics for rural and urban residents. The statistical procedures yielded population-weighted estimates and demonstrated the distributions of demographics and healthcare needs, enabling factors for those dwelling in urban or in rural areas.

We hypothesized that expenditures would be higher for rural populations than for urban populations, possibly due to a greater prevalence of poor health status in rural populations [22], or due to inferior access to (or quality of) preventative care in rural areas [23]. Based on the findings of two-part models, there was no difference in total health expenditures (including or excluding zero users) between rural-urban residents. Although a higher proportion of urban residents had zero total health expenditures, after adjusting 


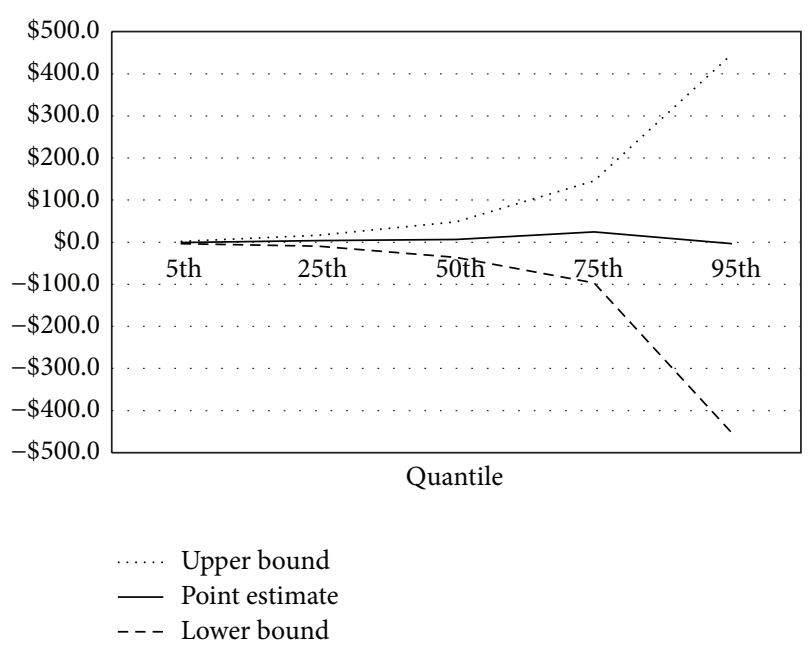

FIGURE 4: Weighted relationships between residence and nonzero expenditure of prescription drug, adjusted by 10 covariates.
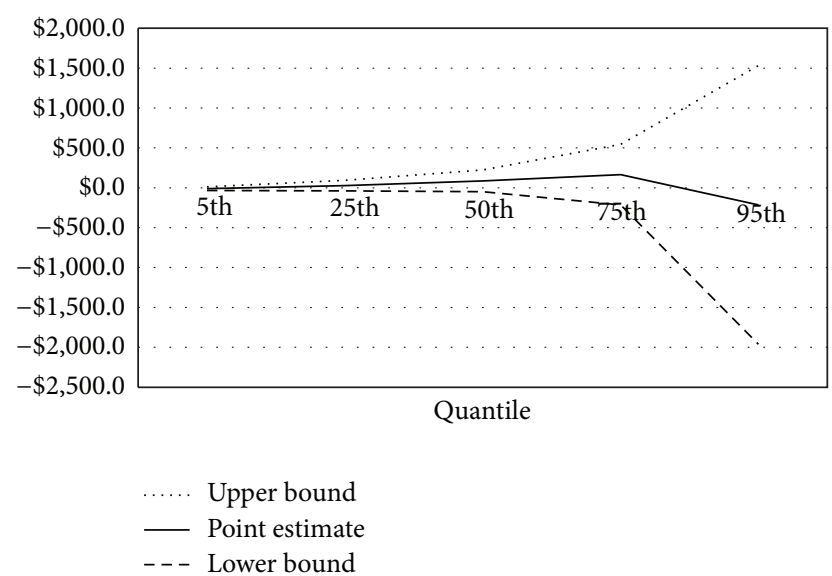

FIGURE 5: Weighted relationships between residence and nonzero total expenditure, adjusted by 10 covariates.

for personal characteristics of rural and urban residents, there was no significant rural-urban difference in the likelihood of zero total expenditure. It suggests that policy makers should look beyond total expenditure when comparing rural-urban differences in health care expenditure.

Expenditures of four different types of health expenditures were also compared between rural and urban populations. Higher proportions of rural dwellers were likely to exhibit expenditures (versus no expenditures) for hospital emergency room services and prescription drugs, though only the prescription drugs differential was statistically significant after adjusting for personal characteristics. However, the urban residents using emergency care services had higher emergency care expenditures than the rural residents who used emergency services.

Rural residents had significantly higher expenditures for prescription medications than their urban counterparts. The MEPS only provides the overall payment information rather than identifying whether pharmacies prescribe and/or fill the prescriptions. However, this result probably is consistent with findings in previous studies that rural residents heavily rely on local pharmacies for keeping them healthy [3, 24, 25]. Since rural adults aged 18 and older in this study were generally older and less educated, had loser income, and were more reliant on public insurance and in poorer health, the rural populations were found to have higher out-of-pocket expenditures on prescription medications $[5,25,26]$.

Employing the quantile regression model is the major extension of previous work. The quantile regression results suggested that the impact of urban-rural residency status might be more pronounced at the highest expenditure quantiles. The trend toward a positive impact of rural residency on expenditures for prescription drugs at higher expenditure quantiles is consistent with the findings of two-part models as well as past studies [27]. These results imply that even looking beyond subcategories of health expenditures may not tell the entire story of differences in expenditures between those dwelling in urban and rural areas. Using methods that allow the investigator to compare these different areas among residents with different levels of health care expenditures may also be enlightening.

4.2. Limitations. Selecting MEPS as the data source had several limitations for addressing our research questions. First of all, Franco pointed out that one-quarter of rural home care users were served by an urban agency and 3\% of urban residents were served by a rural agency [28]. Nevertheless, the MEPS did not identify location of providers or the distances between users' homes and providers. Likewise, detailed information about physicians' referral patterns, hospital characteristics, and county characteristics were found to influence choice of healthcare providers [29, 30]. But the MEPS data set provides no information to assess the associations of these factors with health care expenditures. Further studies are needed to address these issues.

Second, this study only focuses on healthcare expenditures of four types of services used by noninstitutionalized adults aged 18 and older. It is inappropriate to employ the research findings to interpret other kinds of health services (e.g., dental care) and other age groups (e.g., newborns). Research about healthcare utilization/expenditures has gathered increasing attention in recent years [31]. More comparative studies using the MEPS to analyze other types of healthcare expenses and to include other age groups are highly recommended.

\section{Conflict of Interests}

The authors declare that there is no conflict of interests regarding the publication of this paper.

\section{References}

[1] Center for Medicare \& Medicaid Services (CMS), "National health expenditures; aggregate and per capita amounts, annual percent change and percent distribution: selected calendar 
year 1960-2011," 2012, http://www.cms.gov/Research-StatisticsData-and-Systems/Statistics-Trends-and-Reports/NationalHealthExpendData/Downloads/tables.pdf.

[2] CMS, "National health expenditure projections 2011-2021," 2012, http://www.cms.gov/Research-Statistics-Data-and-systems/Statistics-Trends-and-Reports/NationalHealthExpendData/Downloads/Proj2011PDF.pdf.

[3] W. Hawk, "Expenditures of urban and rural households in 2011," 2013, http://www.bls.gov/opub/btn/volume-2/expenditures-ofurban-and-rural-households-in-2011.htm.

[4] J. W. Cohen, A. C. Monheit, K. M. Beauregard et al., "The medical expenditure panel survey: a national health information resource," Inquiry, vol. 33, no. 4, pp. 373-389, 1996.

[5] E. C. Ziller, A. F. Coburn, and A. E. Yousefian, "Out-of-pocket health spending and the rural underinsured," Health Affairs, vol. 25, no. 6, pp. 1688-1699, 2006.

[6] R. J. Manski, H. S. Goodman, B. C. Reid, and M. D. Macek, "Dental insurance visits and expenditures among older adults," American Journal of Public Health, vol. 94, no. 5, pp. 759-764, 2004.

[7] F. M. Chevarley, P. L. Owens, M. W. Zodet, L. A. Simpson, M. C. McCormick, and D. Dougherty, "Health care for children and youth in the United States: annual report on patterns of coverage, utilization, quality, and expenditures by a county level of urban influence," Ambulatory Pediatrics, vol. 6, no. 5, pp. 241264, 2006.

[8] A. N. West and W. B. Weeks, "Health care expenditures for urban and rural veterans in veterans health administration care," Health Services Research, vol. 44, no. 5, pp. 1718-1734, 2009.

[9] K. D. Bertakis, R. Azari, L. J. Helms, E. J. Callahan, and J. A. Robbins, "Gender differences in the utilization of health care services," Journal of Family Practice, vol. 49, no. 2, pp. 147-152, 2000.

[10] P. Diehr, D. Yanez, A. Ash, M. Hornbrook, and D. Y. Lin, "Methods for analyzing health care utilization and costs," Annual Review of Public Health, vol. 20, pp. 125-144, 1999.

[11] J. Pasic, J. Russo, and P. Roy-Byrne, "High utilizers of psychiatric emergency services," Psychiatric Services, vol. 56, no. 6, pp. 678684, 2005.

[12] M. von Korff, J. Ormel, W. Katon, and E. H. B. Lin, "Disability and depression among high utilizers of health care: a longitudinal analysis," Archives of General Psychiatry, vol. 49, no. 2, pp. 91-100, 1992.

[13] Agency for Healthcare Research and Quality (AHRQ), MEPS-HC Panel Design and Data Collection Process, 2013, http://meps.ahrq.gov/mepsweb/survey_comp/hc_data_collection.jsp.

[14] Center for Disease Control and Prevention (CDC), 2010 National Healthcare Quality and Disparities Reports, 2011, http://www.ahrq.gov/research/findings/nhqrdr/nhqrdr10/ datasources/cdc.html.

[15] AHRQ, MEPS HC-138: 2010 Full Year Consolidated Data File, Published 2012. Accessed April 26, 2013, http:// meps.ahrq.gov/data_stats/download_data_files_detail.jsp?cboPufNumber $=$ HC-138.

[16] CDC, "About the National Health Interview Survey," 2013, http://www.cdc.gov/nchs/nhis/about_nhis.htm.

[17] J. T. Spotila, "Standards for defining metropolitan and micropolitan areas: notice," 2000, http://www.whitehouse.gov/sites/ default/files/omb/fedreg/metroareas122700.pdf.
[18] Office of Rural Health Policy (ORHP), "Definition of Rural," 2012, http://www.hrsa.gov/ruralhealth/policy/definition_of_rural.html.

[19] R. M. Andersen, "Revisiting the behavioral model and access to medical care: does it matter?" Journal of Health and Social Behavior, vol. 36, no. 1, pp. 1-10, 1995.

[20] R. Andersen and J. F. Newman, "Societal and individual determinants of medical care utilization in the United States," Milbank Memorial Fund Quarterly. Health \& Society, vol. 51, no. 1, pp. 95-124, 1973.

[21] StataCorp, Stata Statistical Software: Release 12, StataCorp LP, Station, Tex, USA, 2011.

[22] R. A. Crosby, M. L. Wendel, R. C. Vanderpool, and B. R. Casey, Rural Populations and Health, John Wiley \& Sons, San Francisco, Calif, USA, 2012.

[23] J. N. Laditka, S. B. Laditka, B. Olatosi, and K. T. Elder, "The health trade-off of rural residence for impaired older adults: longer life, more impairment," Journal of Rural Health, vol. 23, no. 2, pp. 124-132, 2007.

[24] T. D. McBride, "Why are health care expenditures increasing and is there a rural differential?" Rural Policy Brief, vol. 10, no. 7, pp. 1-8, 2005.

[25] National Economic Council, "Prescription drug coverage \& rural Medicare beneficiaries: a critical unmet need," 2005, http://clinton4.nara.gov/media/pdf/rural_report_6-13.pdf.

[26] C. Caplan and N. Brangan, "Prescription drug spending and coverage among rural Medicare beneficiaries in 2003," 2004, http://assets.aarp.org/rgcenter/post-import/dd106_rx_ spending.pdf.

[27] UnitedHealth Group, "Modernizing rural health care: coverage, quality and innovation," 2011, http://www.unitedhealthgroup.com/Error/PageNotFound.aspx.

[28] S. J. Franco, Medicare Home Health Care in Rural America. Policy Analysis Brief (No. 1), NORC Walsh Center for Rural Health Analysis, Bethesda, Md, USA, 2004.

[29] M. J. Hall, J. Marsteller, and M. Owings, "Factors influencing rural residents' utilization of urban hospitals," National health statistics reports, vol. 18, no. 31, pp. 1-12, 2010.

[30] M. J. Hall, M. F. Owings, and J. A. Shinogle, "Inpatient care in rural hospitals at the beginning of the 21st century," Journal of Rural Health, vol. 22, no. 4, pp. 331-338, 2006.

[31] Federal Trade Commission and the Department of Justice, "Improving health care: a dose of competition," 2004, https:// www.ftc.gov/reports/healthcare/040723healthcarerpt.pdf. 


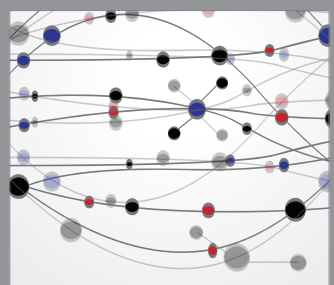

The Scientific World Journal
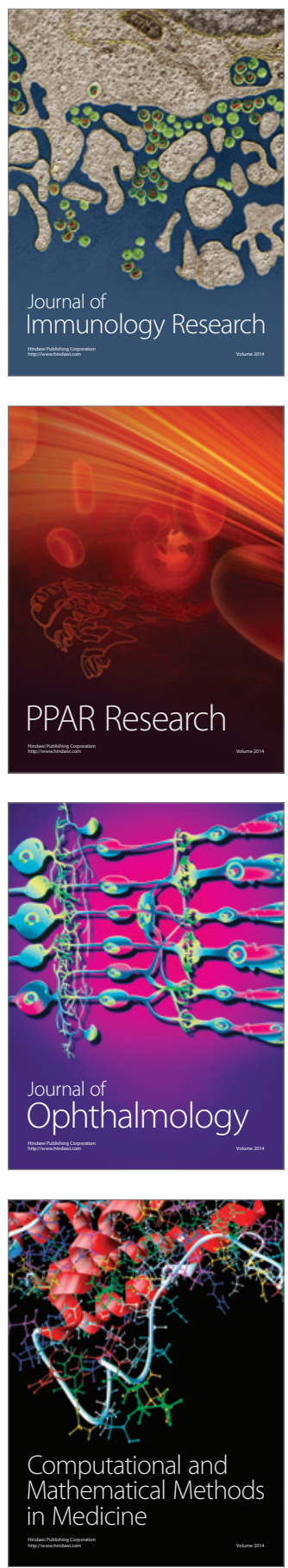

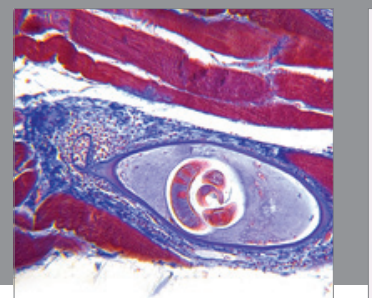

Gastroenterology

Research and Practice
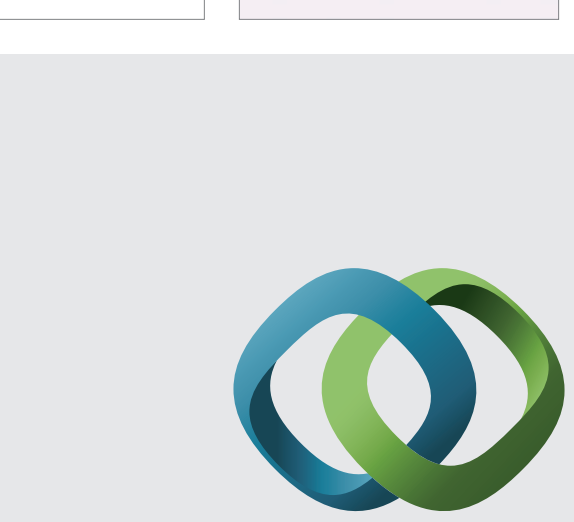

\section{Hindawi}

Submit your manuscripts at

http://www.hindawi.com
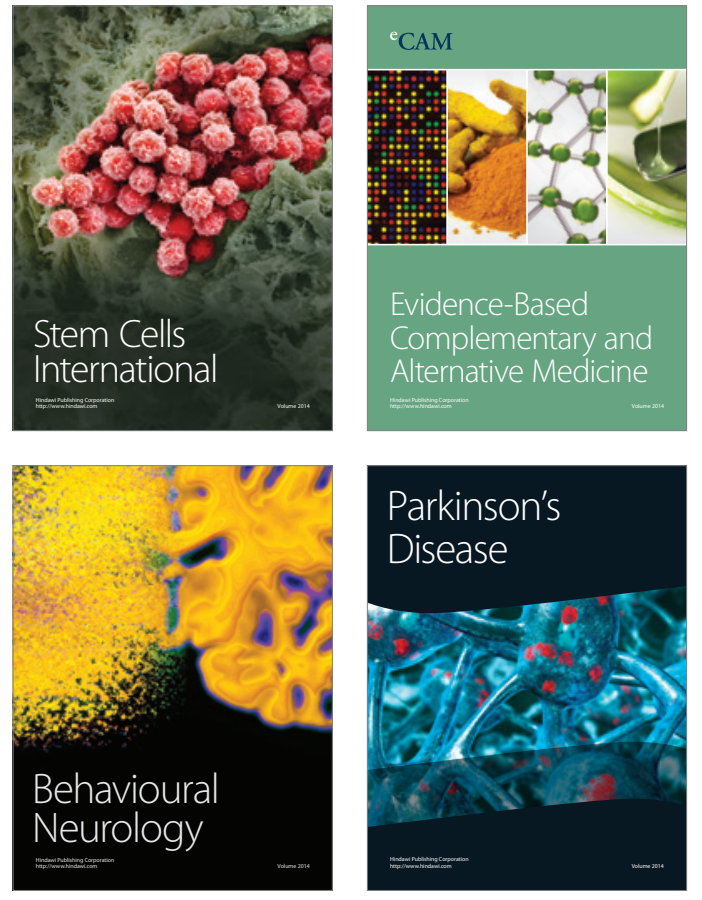
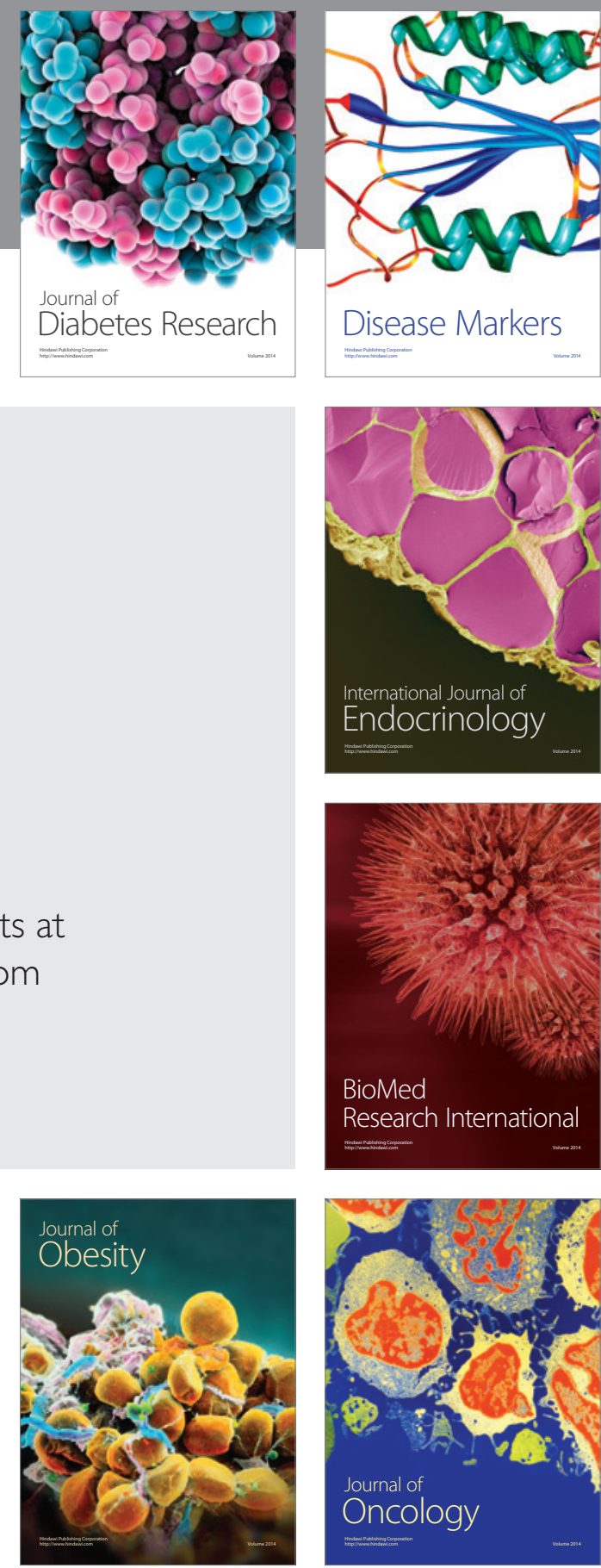

Disease Markers
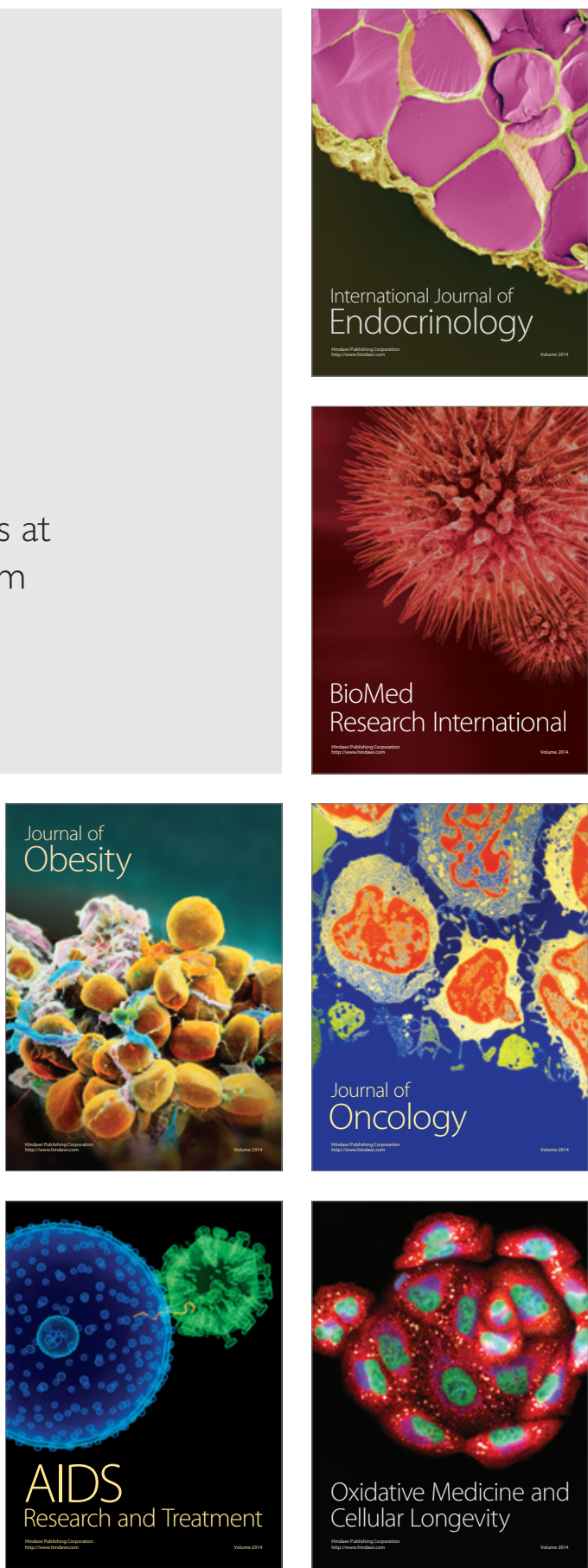